\title{
Does Real World Use of Liraglutide Match its Use in the LEADER Cardiovascular Outcome Trial? Study Protocol
}

\author{
William Hinton $\cdot$ Michael Feher $\cdot$ Neil Munro $\cdot$ \\ Simon de Lusignan
}

Received: January 31, 2018 / Published online: March 31, 2018

(C) The Author(s) 2018

\section{ABSTRACT}

Background: Liraglutide is an injectable therapy to treat type 2 diabetes (T2DM), belonging to the glucagon-like peptide- 1 receptor agonist class of drugs. The Liraglutide Effect and Action in Diabetes: Evaluation of Cardiovascular Outcome Results (LEADER) trial established that liraglutide demonstrated glucose-lowering benefits and improved cardiovascular outcomes in those individuals with T2DM at high cardiovascular risk.

Aims: The aim of this study is to report the prevalence and characteristics of people treated with liraglutide compared with the LEADER trial. In addition, the remaining portion of the T2DM population will be examined to determine the prevalence of those who meet the inclusion criteria for the LEADER trial but who are not treated with this medication.

Study Design and Methods: This is a crosssectional analysis of routinely collected primary care data on all people with T2DM included in the Royal College of General Practitioners

Enhanced content To view enhanced content for this article go to https://doi.org/10.6084/m9.figshare.59092 54 .

W. Hinton · M. Feher - N. Munro ·

S. de Lusignan $(\bowtie)$

Department of Clinical and Experimental Medicine,

University of Surrey, Guildford, UK

e-mail: s.lusignan@surrey.ac.uk
(RCGP) Research and Surveillance Center (RSC) network database. People with T2DM will be identified from the dataset using a well-established ontological process. Read and other clinical codes will be used to identify people prescribed liraglutide and those at high cardiovascular risk. We will use descriptive statistics to report the characteristics of people with T2DM prescribed liraglutide compared with those of the LEADER trial and the proportion of the wider T2DM cohort that matches the LEADER inclusion criteria. In terms of ethical considerations, this study used pseudonymized data, and was classed as an "Audit of current practice".

Planned Outputs: The results of the study will be submitted for publication in a peer-reviewed journal to report the applicability of the results of the LEADER trial to real-world clinical practice.

Funding: Novo Nordisk Limited.

Keywords: Cardiovascular diseases; Crosssectional studies; Diabetes mellitus, type 2; Liraglutide; Medical record systems, computerized

\section{INTRODUCTION}

Liraglutide is an established injectable therapy, belonging to the glucagon-like peptide-1 (GLP1) receptor agonist class of drugs, for the treatment of hyperglycemia in people with type 2 
diabetes (T2DM). Like other medications within its class, liraglutide improves glycemic control by stimulating the GLP-1 receptor to produce more insulin, while simultaneously suppressing the secretion of glucagon and prolonging gastric emptying [1, 2]. Clinical trials have demonstrated that liraglutide has various benefits, including reduced glycated hemoglobin (HbA1c), decreased risk of hypoglycemia compared with sulfonylurea, and weight loss $[3,4]$. Liraglutide has also been shown to have renoprotective effects and to reduce systolic blood pressure [5-7]. More recently, the effects of liraglutide have been explored in a cardiovascular outcome trial (CVOT).

CVOTs are safety trials for the new classes of drugs used in the management of T2DM. The Liraglutide Effect and Action in Diabetes: Evaluation of Cardiovascular Outcome Results (LEADER) study was a double-blind randomized controlled trial assessing the cardiovascular effects of liraglutide compared to a placebo, when added to standard care in patients with T2DM [8]. It was also designed to evaluate any inferiority or superiority in terms of cardiovascular benefit.

The study comprised 9340 individuals with T2DM and high cardiovascular disease risk. After a median follow-up of 3.8 years, time-toevent analysis confirmed the liraglutide was associated with a lower rate of first occurrence of death from cardiovascular causes, nonfatal myocardial infarction, or nonfatal stroke compared with placebo. The results of the LEADER trial confirmed not only the non-inferiority of liraglutide with respect to cardiovascular safety but provided evidence of its superiority in terms of cardiovascular outcomes.

Whether these findings translate into realworld clinical practice is unclear. Here we set out the method we will use to determine (1) the current pattern of prescribing liraglutide within a nationally representative general practice network and (2) the prevalence of people with T2DM possessing the same cardiovascular risk profile as those included in the LEADER trial. This will inform the extent to which the findings of the LEADER trial can be generalized to routine clinical practice.

\section{AIMS AND METHODS}

We will perform a cross-sectional analysis of all people with T2DM included in the Royal College of General Practitioners (RCGP) Research and Surveillance Center (RSC) database to identify people prescribed liraglutide and to describe their cardiovascular risk profile. In addition, the proportion of those with a cardiovascular risk profile comparable to that of those included in the LEADER trial will be reported.

\section{Objectives}

The aim of the study is to compare the clinical characteristics of patients prescribed liraglutide with those people included in the LEADER trial. We also aim to identify the proportion of patients in the RCGP RSC database that meet the inclusion criteria for liraglutide as used in the LEADER trial but who are not prescribed this medication.

The primary objectives are:

1. To identify the number of people prescribed liraglutide in UK primary care who meet the inclusion criteria for the LEADER trial.

2. To describe the characteristics of patients taking liraglutide:

a. The number of people that meet each of the LEADER inclusion criteria.

b. The duration of their diabetes.

c. The number of people identified on concurrent oral antihyperglycemic medications or on insulin.

3. To describe the demographic (age, gender, ethnicity, socioeconomic status $\{$ SES, using the Index of Multiple Deprivation [9]\}) and clinical characteristics (HbA1c, blood pressure, renal function, body mass index, etc.) of people in each of the groups described above.

The secondary objectives are:

4. To identify the number of people in the entire RCGP RSC cohort who meet the inclusion criteria for the LEADER trial.

5. To describe the characteristics of the people with T2DM eligible for the LEADER trial: 
a. The number of people who meet each of the LEADER inclusion criteria.

b. The duration of their diabetes.

c. The number of those identified on concurrent oral antihyperglycemic medications or on insulin.

6. To describe the demographic (age, gender, ethnicity, SES) and clinical characteristics (HbA1c, blood pressure, renal function, body mass index, etc.) of people in each of the groups described above.

\section{Data Source}

The RCGP RSC is a primary care sentinel network that includes the records from over 200 primary care practices distributed across England covering a population of over 2,000,000 patients. This is a nationally representative sample [10], primarily used for the monitoring of respiratory disease and infections such as influenza for vaccine effectiveness [11-13].

UK general practice is suited to this type of study because it is a registration-based system; each patient is registered with a single practice and if they move their data move with them [14]. Each patient also has a unique patient identifier, the National Health Service (NHS) number, which not only facilitates specific patient-data linkage but also the potential for data linkage with other datasets, such as pathology results. Repeat prescription data have been complete since the 1990s following the transition from paper-based to computerized medical records (CMR) in UK primary care, while the coding of chronic disease and laboratory links were standardized in 2004 [15]. We have recently demonstrated our ability to monitor adherence to different classes of therapies using this database [16].

UK primary care CMR data are recorded using the Read classification [17]. The coded data includes patient demographics (age, sex, ethnicity, and SES), diagnosis and processes of care (referrals, annual reviews, care pathways, etc.), prescriptions, and laboratory data. Inclusion of data recording targets in the UK primary care pay-for-performance targets have led to a high level of data completeness in these records, particularly in the population of people with T2DM [10].

We will use all data collected from primary care practices after 1 January 2016, which will comprise all patients with a T2DM diagnosis who are over the age of 18 years. Within this cohort, we will identify and report the proportion of those with a prescription for liraglutide, and all of those with similar cardiovascular risk/conditions to those of the LEADER trial. The demographic and clinical characteristics of these groups will be reported. Missing data for each variable of interest will also be stated.

In the interests of information governance, the RCGP RSC data is pseudonymized by NHS number. This study was classed as an "Audit of current practice," and therefore specific ethical approval was not required.

\section{Data Analysis}

People with T2DM will be identified using a two-step process that we have reported in detail elsewhere [18]. This is an ontological-based approach that integrates numerous data elements to enhance case definition [19]. Initially, all people with diabetes are identified using a combination of diagnostic codes, HbA1c and blood glucose test results (two or more that confirm diabetes), and medication use (except metformin). Codes specific to gestational diabetes or other types of secondary diabetes are excluded. People are then categorized by diabetes type (T1DM, T2DM, undetermined diabetes type) using a seven-step algorithm that takes into account previous and current drug therapies used, diagnosis codes specific to diabetes type, and other important clinical characteristics (age and body mass index at diagnosis, and duration of oral anti-hyperglycemic medications).

We will use the high cardiovascular risk inclusion criteria for the LEADER trial to determine prevalence within the T2DM cohort (Table 1). People will be identified with each cardiovascular risk factor using the most similar diagnostic codes, or other codes which determine diagnosis of a risk factor. We will provide a 
Table 1 Inclusion criteria for the LEADER trial taken from the supplementary material provided in the LEADER publication by Marso et al. [8]

\section{LEADER trial inclusion criteria}

T2DM with $\mathrm{HbA} 1 \mathrm{c} \geq 7 \%$

Cardiovascular disease: $\geq 50$ years of age and $\geq 1$ of the following:

Previous myocardial infarction

Previous stroke or transient ischemic attack

Previous coronary, carotid or peripheral arterial revascularization

$>50 \%$ stenosis of coronary, carotid, or lower extremity arteries

History of symptomatic coronary heart disease documented by positive exercise stress test or any cardiac imaging or unstable angina with ECG changes

Asymptomatic cardiac ischemia documented by positive nuclear imaging test, exercise test or dobutamine stress echo

Chronic heart failure New York Heart Association class II-III

Chronic renal failure:

eGFR $<60 \mathrm{~mL} / \mathrm{min} / 1.73 \mathrm{~m}^{2}$ (Modification of

Diet in Renal Disease formula)

eGFR $<60 \mathrm{~mL} / \mathrm{min}$ (Cockcroft-Gault formula)

No previous cardiovascular disease group: $\geq 60$ years and $\geq 1$ of the following:

Microalbuminuria (ACR) or proteinuria

Hypertension and left ventricular hypertrophy by ECG or imaging

Left ventricular systolic or diastolic dysfunction by imaging

Ankle-brachial index $<0.9$

T2DM Type 2 diabetes mellitus, HbAlc glycated hemoglobin, $E C G$ electrocardiogram, $e G F R$ estimated glomerular filtration rate, $A C R$ albumin:creatinine ratio complete list of codes and a full description of the process in the final manuscript.

\section{Statistical Methods}

We will use descriptive statistics (percentages, means, standard deviations, etc.) to describe the characteristics of the cohorts. Crude rates of each variable of interest will be reported within each cohort, with 95\% confidence intervals.

\section{STRENGTHS AND LIMITATIONS}

A number of strengths of the dataset have been described in the section "Data Source". The large denominator ( $>2$ million patients) of this high-quality real-world evidence dataset is a specific strength. We have also previously demonstrated our ability to compare real-world and trial use of another class of medication in diabetes [20], and also to link complex data, in this example pancreatitis, to cases of diabetes [21]. However, given that the minority of people with T2DM are prescribed liraglutide, there may not be enough statistical power to confirm "true differences" between groups.

Another potential limitation is missing data in a patient's primary care record, whereby a number of patients may have particular conditions that have not been recorded. Nonetheless, the pay for performance scheme (P4P), i.e., the Quality and Outcomes Framework (QOF), implemented in 2004 to incentivize primary care practices to achieve indicator thresholds for the management of chronic diseases [22], has improved data quality in primary care. Additional strengths and limitations found while undertaking the study will be reported in the final version of the manuscript.

\section{CONCLUSION}

This real-world evidence cross-sectional analysis will investigate the prevalence of people with T2DM in a nationally representative primary care population currently prescribed liraglutide and their cardiovascular risk profile; and those 
in the wider T2DM population that meet the inclusion criteria for the LEADER trial.

\section{ACKNOWLEDGEMENTS}

The authors would like to thank patients and practices who are members of the RCGP RSC network, and the CMR system vendors: EMIS, In Practice, and TPP. We would also like to thank Apollo Medical systems, the RCGP, and University of Surrey colleagues: Filipa Ferreira (Programme Manager), Rachel Byford, and Julian Sherlock (SQL developers).

Funding. Sponsorship for this study and article processing charges were funded by Novo Nordisk Limited. All authors will have full access to all of the data in this study and will take complete responsibility for the integrity of the data and accuracy of the data analysis.

Authorship. All named authors meet the International Committee of Medical Journal Editors (ICMJE) criteria for authorship for this manuscript, take responsibility for the integrity of the work as a whole and have given final approval for the version to be published.

Authorship Contributions. William Hinton led the drafting of the manuscript. Simon de Lusignan, Michael Feher, and Neil Munro reviewed the manuscript and study design and contributed to the final manuscript draft.

Disclosures. William Hinton receives research funding from Eli Lilly and Co. and Novo Nordisk Ltd. Simon de Lusignan receives research funding from Eli Lilly Co., GlaxoSmithKline, Takeda, AstraZeneca, and Novo Nordisk Ltd. Neil Munro has received financial support for research, speaker meetings and consultancy from MSD, Merck, BMS, AstraZeneca, Pfizer, Novo Nordisk Ltd, Eli Lilly and Co., and Sanofi-Aventis. Michael Feher also receives financial support for research, speaker meetings and consultancy from MSD, Merck, BMS, AstraZeneca, Pfizer, Novo Nordisk Ltd, Eli Lilly and Co., and Sanofi-Aventis.
Compliance with Ethics Guidelines. This study is considered to be an "Audit of current practice" when tested against the Health Research Authority (HRA)/Medical Research Council (MRC) "Is my study research" tool and therefore did not require specific ethical approval [23]. In addition, the processes and systems are streamlined to operationalize these studies, as we consistently strive to follow best practices in surveillance and quality improvement $[24,25]$. Approval for use of the data was acquired from the RCGP RSC Study Approval Committee.

Open Access. This article is distributed under the terms of the Creative Commons Attribution-NonCommercial 4.0 International License (http://creativecommons.org/licenses/ by-nc/4.0/), which permits any noncommercial use, distribution, and reproduction in any medium, provided you give appropriate credit to the original author(s) and the source, provide a link to the Creative Commons license, and indicate if changes were made.

\section{REFERENCES}

1. Baggio LL, Drucker DJ. Biology of incretins: GLP-1 and GIP. Gastroenterology. 2007;132(6):2131-57.

2. Nice Institute for Health Care Excellence. Liraglutide. Available at: https://bnf.nice.org.uk/drug/ liraglutide.html. Accessed 10 Jan 2018.

3. Kim SH, Abbasi F, Lamendola C, et al. Benefits of liraglutide treatment in overweight and obese older individuals with prediabetes. Diabetes Care. 2013;36(10):3276-82.

4. Parks M, Rosebraugh C. Weighing risks and benefits of liraglutide-the FDA's review of a new antidiabetic therapy. N Engl J Med. 2010;362(9):774-7.

5. Goud A, Zhong J, Peters M, Brook RD, Rajagopalan S. GLP-1 agonists and blood pressure: a review of the evidence. Curr Hypertens Rep. 2016;18(2):16.

6. Sun F, Wu S, Guo S, et al. Impact of GLP-1 receptor agonists on blood pressure, heart rate and hypertension among patients with type 2 diabetes: a systematic review and network meta-analysis. Diabetes Res Clin Pract. 2015;110(1):26-37. 
7. von Scholten BJ, Persson F, Rosenlund S, et al. The effect of liraglutide on renal function: a randomized clinical trial. Diabetes Obes Metab. 2017;19(2):239-47.

8. Marso SP, Daniels GH, Brown-Frandsen K, et al. Liraglutide and cardiovascular outcomes in type 2 diabetes. N Engl J Med. 2016;375(4):311-22.

9. GOV.UK. English indices of deprivation 2015. Available at: https://www.gov.uk/government/ statistics/english-indices-of-deprivation-2015. Accessed 17 Jan 2018.

10. Correa A, Hinton W, McGovern A, et al. Royal College of General Practitioners Research and Surveillance Centre (RCGP RSC) sentinel network: a cohort profile. BMJ Open. 2016;6(4):e011092.

11. Fleming DM, Miles J. The representativeness of sentinel practice networks. J Public Health (Oxf). 2010;32(1):90-6.

12. Fleming DM, Schellevis FG, Paget WJ. Health monitoring in sentinel practice networks: the contribution of primary care. Eur J Public Health. 2003;13[3 Suppl]:80-4.

13. Queenan JA, Williamson T, Khan S, et al. Representativeness of patients and providers in the Canadian Primary Care Sentinel Surveillance Network: a cross-sectional study. CMAJ Open. 2016;4(1):E28.

14. de Lusignan S, van Weel C. The use of routinely collected computer data for research in primary care: opportunities and challenges. Fam Pract. 2006;23(2):253-63.

15. de Lusignan S, Metsemakers JF, Houwink P, Gunnarsdottir V, van der Lei J. Routinely collected general practice data: goldmines for research? A report of the European Federation for Medical Informatics Primary Care Informatics Working Group (EFMI PCIWG) from MIE2006, Maastricht, The Netherlands. Inform Prim Care. 2006;14(3):203-9.

16. McGovern A, Hinton W, Calderara S, Munro N, Whyte $M$, de Lusignan S. A class comparison of medication persistence in people with type 2 diabetes: a retrospective observational study. Diabetes Ther. 2018;9(1):229-42.
17. de Lusignan S. Codes, classifications, terminologies and nomenclatures: definition, development and application in practice. Inform Prim Care. 2005; 13(1):65-70.

18. McGovern A, Hinton W, Correa A, Munro N, Whyte $M$, de Lusignan S. Real-world evidence studies into treatment adherence, thresholds for intervention and disparities in treatment in people with type 2 diabetes in the UK. BMJ Open. 2016;6(11):e012801.

19. Liaw ST, Taggart J, Yu H, de Lusignan S, Kuziemsky $\mathrm{C}$, Hayen A. Integrating electronic health record information to support integrated care: practical application of ontologies to improve the accuracy of diabetes disease registers. J Biomed Inform. 2014;52:364-72.

20. McGovern A, Feher M, Munro N, de Lusignan S. Sodium-glucose co-transporter 2 (SGLT2) inhibitor: comparing trial data and real-world use. Diabetes Ther. 2017;8(2):365-76.

21. Woodmansey C, McGovern AP, McCullough KA, et al. Incidence, demographics, and clinical characteristics of diabetes of the exocrine pancreas (type $3 c)$ : a retrospective cohort study. Diabetes Care. 2017;40(11):1486-93.

22. Langdown C, Peckham S. The use of financial incentives to help improve health outcomes: is the quality and outcomes framework fit for purpose? A systematic review. J Public Health (Oxf). 2014;36(2):251-8.

23. Health Research Authority. Is my study research? Available at:http://www.hra-decisiontools.org.uk/ research/. Accessed 17 Jan 2018.

24. Chan T, Di Iorio CT, De Lusignan S, Lo Russo D, Kuziemsky C, Liaw ST. UK National Data Guardian for Health and Care's Review of Data Security: trust, better security and opt-outs. J Innov Health Inform. 2016;23(3):627-32.

25. De Lusignan $\mathrm{S}$, Liyanage $\mathrm{H}$, Di Iorio CT, Chan $\mathrm{T}$, Liaw ST. Using routinely collected health data for surveillance, quality improvement and research: framework and key questions to assess ethics, privacy and data access. J Innov Health Inform. 2016;22(4):426-32. 